\title{
Terascale Simulations for Heavy Ion Inertial Fusion Energy
}

A. Friedman, R. H. Cohen, D. P. Grote, W. M. Sharp, C. M. Celata, E. P. Lee, J-L. Vay, R. C. Davidson, I. Kaganovich, W. W. Lee, H. Qin, D. R. Welch, I. Haber, R. A. Kishek

\section{June 8, 2000}

U.S. Department of Energy

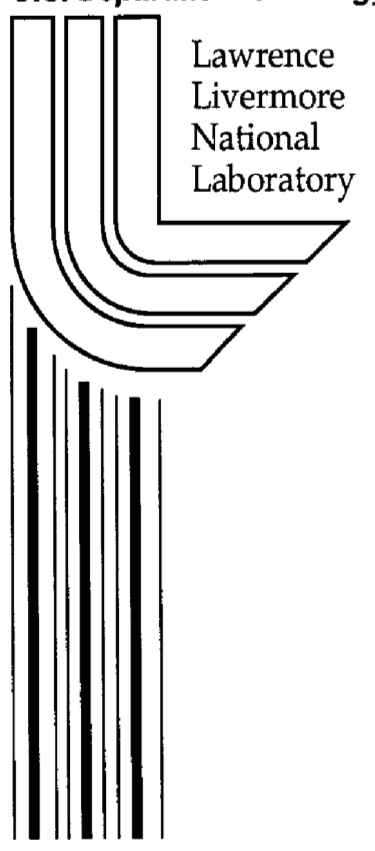




\section{DISCLAIMER}

This document was prepared as an account of work sponsored by an agency of the United States Government. Neither the United States Government nor the University of California nor any of their employees, makes any warranty, express or implied, or assumes any legal liability or responsibility for the accuracy, completeness, or usefulness of any information, apparatus, product, or process disclosed, or represents that its use would not infringe privately owned rights. Reference herein to any specific commercial product, process, or service by trade name, trademark, manufacturer, or otherwise, does not necessarily constitute or imply its endorsement, recommendation, or favoring by the United States Government or the University of California. The views and opinions of authors expressed herein do not necessarily state or reflect those of the United States Government or the University of California, and shall not be used for advertising or product endorsement purposes.

This work was performed under the auspices of the U. S. Department of Energy by the University of California, Lawrence Livermore National Laboratory under Contract No. W-7405-Eng-48.

This report has been reproduced directly from the best available copy.

Available to DOE and DOE contractors from the Office of Scientific and Technical Information

P.O. Box 62, Oak Ridge, TN 37831

Prices available from (423) 576-8401 http://apollo.osti.gov/bridge/

Available to the public from the National Technical Information Service

U.S. Department of Commerce 5285 Port Royal Rd., Springfield, VA 22161 http://www.ntis.gov/

OR

Lawrence Livermore National Laboratory Technical Information Department's Digital Library http://www.llnl.gov/tid/Library.html 


\title{
TERASCALE SIMULATIONS FOR HEAVY ION INERTIAL FUSION ENERGY*
}

A. Friedman, R. H. Cohen, D. P. Grote, W. M. Sharp (LLNL); C. M. Celata, E. P. Lee, J-L. Vay (LBNL); R. C. Davidson, I. Kaganovich, W. W. Lee, H. Qin (PPPL); D. R. Welch (MRC);

\section{Proposed Research}

\author{
I. Haber (NRL); R. A. Kishek (U. Md.)
}

The intense ion beams in a heavy ion Inertial Fusion Energy (IFE) driver and fusion chamber are non-neutral plasmas whose dynamics are largely dominated by space charge. We propose to develop a "source-to-target" Heavy Ion Fusion (HIF) beam simulation capability: a description of the kinetic behavior of this complex, nonlinear system which is both integrated and detailed. We will apply this new capability to further our understanding of key scientific issues in the physics of ion beams for IFE. The simulations will entail self-consistent field descriptions that require interprocessor communication, but are scalable and will run efficiently on terascale architectures.

This new capability will be based on the integration of three types of simulations, each requiring terascale computing: (1) simulations of acceleration and confinement of the space-charge-dominated ion beams through the driver (accelerator, pulse compression line, and final focusing system) which accurately describe their dynamics, including emittance growth (phase-space dilution) effects; these are particle-in-cell (PIC) models; (2) electromagnetic (EM) and magnetoinductive (Darwin) simulations which describe the beam and the fusion chamber environment, including multibeam, neutralization, stripping, beam and plasma ionization processes, and return current effects; and (3) highly detailed simulations ( $\delta \mathrm{f}$, multispecies PIC, continuum Vlasov), which can examine electron effects and collective modes in the driver and chamber, and can study halo generation with excellent statistics, to ensure that these effects do not disrupt the focusability of the beams.

The code development will involve: (i) adaptation of existing codes to run efficiently on multi-SMP computers that use a hybrid of shared and distributed memory; (ii) development of new and improved numerical algorithms, e.g., averaging techniques that will afford larger timesteps; and (iii) incorporation of improved physics models (e.g., for self-magnetic, module impedance, atomic physics, and multibeam effects) that will be made practical by the terascale capability. The codes will be linked using scripting tools for intercommunication and code steering, "workspace" tools for heterogeneous computations, and self-describing data files (e.g., NetCDF). Graphical output issues will also be addressed; indeed the "data glut" from such simulations, and the time-dependent 6-D phase space involved, are key considerations. A qualitative increase in capability is proposed, as shown in the following table:

\begin{tabular}{|l|l|}
\hline \multicolumn{1}{|c|}{ Present day } & \multicolumn{1}{|c|}{ End of proposal period } \\
\hline $\begin{array}{l}\text { End-to-end 2-D simulations of next-generation } \\
\text { experiments; 3-D of parts of driver; run linking }\end{array}$ & $\begin{array}{l}\text { Integrated 3-D particle simulations of driver } \\
\text { from source to target; multibeam effects }\end{array}$ \\
\hline $\begin{array}{l}\text { Chamber propagation studies using axisymmetric } \\
\text { electromagnetic particle codes }\end{array}$ & $\begin{array}{l}\text { Chamber propagation in 3-D; linkage from } \\
\text { driver simulation; multibeam effects }\end{array}$ \\
\hline $\begin{array}{l}\text { Studies of halos and beam-plasma instabilities using } \\
\text { idealized initial conditions }\end{array}$ & $\begin{array}{l}\text { Linking of end-to-end run data into halo and } \\
\text { instability calculations to ensure fidelity }\end{array}$ \\
\hline
\end{tabular}

\section{Scientific and Technical Benefit}

The physics of the intense ion beams needed for IFE is both rich and subtle, due to the kinetic and nonlinear nature of the system, and the wide range in spatial and temporal scales involved. Focusing onto the target imposes strict constraints, and so emittance growth due to both instabilities and non-ideal processes must be understood. The beams must propagate through the fusion chamber and onto the target with the required brightness and pulse shape. Ionization of background gas, and beam stripping, neutralization, and photoionization by $\mathrm{x}$-rays from the target, are all important, as are potential filamentation and two-stream instabilities. The 3-D chamber calculations proposed here will, for the first time, offer a realistically complete model of the chamber environment, with which various chamber propagation modes can be explored. They will also employ multiple models, and we will compare, e.g., implicit EM and Darwin methods (which can eliminate fast timescales not essential to the physics) against explicit EM methods. In the accelerator, the beam dynamics is nearly collisionless and Liouvillean, and so emittance growth primarily takes place through complicated distortions, driven by collective behaviors, imperfect applied fields, image fields from nearby conductors, and inter-beam forces. The qualitatively improved tools to be developed will lead to a much deeper understanding of these processes.

The above-mentioned effects have been simulated approximately and only for isolated segments of the system. It has been impossible to model the complete system self-consistently, so that the beam distribution function is carried through an end-to-end simulation of the accelerator and into the fusion

* This work was performed under the auspices of the U.S. Department of Energy by the University of California Lawrence Livermore and Lawrence Berkeley National Laboratories under Contract Numbers W-7405-Eng-48 and DE-AC03-76SF00098. 
chamber. Collisionless beams have a "long memory," and initialization of a beam at mid-system with an idealized particle distribution introduces uncertainties. With improved codes on terascale facilities, the full problem can at last be tackled with the expectation of success.

\section{Readiness for Terascale Computing}

The principal codes to be employed are well positioned to move quickly to a terascale platform. The majority of the proposed simulations are between one and two orders of magnitude beyond current practice; in the terascale environment we anticipate typical run times of order a day.

The 3-D PIC code WARP, developed at LLNL and NRL, is used extensively throughout the HIF program for studies of beams in the accelerator, pulse-compression line, and final focusing optic. WARP runs in parallel on NERSC's Cray T3E-900, using message passing. Good scaling has been obtained using up to 256 processors. For full driver runs, many simulation particles (of order 100 million), and at least a hundred grid cells transversely, and thousands longitudinally, will be needed. The timesteps used must be small relative to the beam residence time, because variations in the applied fields must be resolved; thus a driver simulation will require of order 100,000 steps. A run using of order 100 million particles can be expected to take $\sim 2$ days on a 5 Teraflop computer.

Electromagnetic particle codes simulating the beam and the chamber plasma are in use. These include BPIC (Orsay and LBNL; $r, z$ and 3-D, parallelized on the T3E) and LSP (MRC; $r z$ and 3-D with implicit particle and fluid models, and a two-level domain decomposition designed for multilevel memory access). BPIC offers time-dependent mesh spacing in 3-D, which is useful for a converging beam; LSP's implicit hybrid model makes it especially suitable for dense-plasma scenarios. In small ( $10^{5}$ cells) initial tests, LSP achieved speedup factors of up to 26 using 32 processors. We will assess these tools' adaptability to terascale problems, and will employ one or both to study pulse-shaped beams, multibeam effects, target charging, inhomogeneous background plasmas, and other important effects.

A 3-D multispecies $\delta f$ code, BEST, is under development at PPPL This code employs an electrostatic model; a Darwin model will be implemented. The code will be used for detailed studies of beam propagation and stability, beam-plasma interaction, halo formation, mismatch, and emittance. Initial conditions will ultimately come from the codes described above. BEST will use domain decomposition, in a design similar to that already in use by the GTC code for tokamaks.

We have just begun exploring the use of continuum Vlasov methods which evolve the beam distribution function on a multi-dimensional grid. A prototype code, SLV, is currently running on simple problems. We propose to explore this method further, and, if it proves promising, develop and use it for detailed simulations, taking advantage of its natural ability to track low-density regions of phase space, i.e., the beam halo region.

\section{Impact on Other Scientific Disciplines}

The proposed capability involves nonlinear dynamics, self-consistent fields, large-scale parallel computations, massive data handling, interactive and script-driven code steering, and advanced visualization of a time-dependent 6-D phase space. These aspects appear in many emerging applications of terascale computing and considerable cross fertilization with other areas can be anticipated.

This research will be coordinated with the MFE elements of this Fusion advanced computing activity. Commonalities include particle advance and field solution in a terascale environment, emerging Vlasov methods, and advanced scientific visualization.

This research will also be coordinated with research into advanced accelerator simulations being initiated in support of High Energy and Nuclear Physics and Basic Energy Sciences goals. Other accelerator applications are moving toward higher currents, and the knowledge gained through this research into extremely strong space charge should have relevance to a wide variety of such applications. PIC codes are now being developed to look at beam-beam effects near the interaction point in, e.g., the BFactory at SLAC. In general, we may anticipate long-term benefits to such important efforts as the Spallation Neutron Source, Next Linear Collider, Accelerator Transmutation of Waste, Muon Collider, Boron Neutron Capture Therapy, Free Electron Lasers, and others.

\section{Proposed Manpower and Resources}

\begin{tabular}{|c|c|c|c|c|}
\hline (per year; staff levels include post-docs and are averages) & Staff & $\$ \mathrm{k} / \mathrm{y}$ & Total Staff & Total $\$ \mathrm{k} / \mathrm{y}$ \\
\hline LBNL (including LLNL staff) subcontracting with: & 2.45 & 350 & \multirow[t]{3}{*}{ 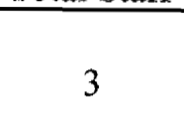 } & \multirow{3}{*}{425} \\
\hline University of Maryland & 0.4 & 50 & & \\
\hline Naval Research Laboratory & 0.15 & 25 & & \\
\hline PPPL, subcontracting with: & 1.25 & 225 & \multirow[t]{2}{*}{1.75} & \multirow{2}{*}{$\overline{325}$} \\
\hline Mission Research Corp & 0.5 & 100 & & \\
\hline TOTAL & 4.75 & $\overline{750}$ & 4.75 & 750 \\
\hline
\end{tabular}

